\title{
Antitumor effects of targeting myeloid-derived suppressive cells
}

\author{
Dong Zeng, Haixia Long, Bo Zhu \\ Institute of Cancer, Xinqiao Hospital, Army Medical University, Chongqing, China \\ Contributions: (I) Conception and design: All authors; (II) Administrative support: H Long and B Zhu; (III) Provision of study materials or patients: \\ All authors; (IV) Collection and assembly of data: All authors; (V) Data analysis and interpretation: All authors; (VI) Manuscript writing: All authors; \\ (VII) Final approval of manuscript: All authors. \\ Correspondence to: Haixia Long, PhD; Bo Zhu, PhD, MD. Institute of Cancer, Xinqiao Hospital, Army Medical University, Chongqing 400037, \\ China. Email: longhaixialhx@163.com; bo.zhu@tmmu.edu.cn.
}

\begin{abstract}
Myeloid-derived suppressor cells (MDSCs) are a heterogeneous population of immature myeloid cells with major regulatory functions, which are expanded in pathological conditions, including cancers, infections and autoimmune diseases. Evidence has identified MDSCs as critical cells driving immune suppression in tumor microenvironments. Treatments targeting MDSCs have shown promising results in preclinical studies and some clinical trials. In this review, we discuss therapeutic approaches targeting MDSCs, which may benefit future study.
\end{abstract}

Keywords: Myeloid-derived suppressor cells (MDSCs); immune; antitumor

Submitted Oct 30, 2019. Accepted for publication Jan 02, 2020.

doi: $10.21037 /$ tcr.2020.01.52

View this article at: http://dx.doi.org/10.21037/tcr.2020.01.52

\section{Introduction}

Myeloid cells from hematopoietic precursor cells mature into terminally differentiated populations, such as dendritic cells, macrophages and granulocytes. However, myelopoiesis is altered under inflammation conditions (1). Myeloid-derived suppressor cells (MDSCs) are a heterogeneous population of immature myeloid cells with major regulatory functions, which are expanded under pathological conditions, including cancers, infections and autoimmune diseases (2). The term MDSCs was first introduced by a group of researchers in 2007, indicating the myeloid origin and immune suppressive function (3). In many cancer types, for example, non-small cell lung cancer (NSCLC), breast cancer, hepatocellular carcinoma, melanoma, multiple myeloma, renal cell carcinoma, prostate cancer and colorectal cancer, MDSCs play essential roles in immunosuppression (4-11). MDSCs are critical cells driving immune suppression in the tumor microenvironment $(12,13)$. MDSCs can also act as biomarkers for evaluating prognoses in tumor patients $(14,15)$. Many studies have targeted MDSCs in preclinical and clinical trials. In this review, we discuss antitumor effects of targeting MDSCs.

\section{Basic information of MDSCs}

Phenotypic and functional characteristics of MDSCs

A detailed review of MDSCs phenotypes has been published (16). In mice, MDSCs are characterized by the expression of CD11b and Gr1. While CD $11 \mathrm{~b}^{+} \mathrm{Gr}-1^{+}$cells are heterogeneous, additional characterization is required. In mice, MDSCs are further divided into monocytic MDSCs (M-MDSCs) and polymorphonuclear MDSCs (PMNMDSCs), having CD11 $\mathrm{b}^{+}$Ly6G Ly6 $\mathrm{C}^{\text {high }}$ and $\mathrm{CD} 11 \mathrm{~b}^{+}$Ly6G ${ }^{+}$ Ly6C ${ }^{\text {low }}$ markers, respectively (16). In human, MDSCs have not been clearly characterized because of their few numbers and the complexity of the myeloid cell system. A research group has proposed human MDSCs populations as $\mathrm{CD}^{-}$ CD $19^{-} \mathrm{CD}_{5} 6^{-} \mathrm{CD} 11 \mathrm{~b}^{+} \mathrm{CD} 33^{+} \mathrm{HLA}^{-} \mathrm{DR}^{-}$. They defined human PMN-MDSCs as CD11 $\mathrm{b}^{+} \mathrm{CD} 14^{-} \mathrm{CD} 15^{+}$or $\mathrm{CD} 11 \mathrm{~b}^{+}$ $\mathrm{CD}_{14} \mathrm{CD}^{-} 6 \mathrm{~b}^{+}$and M-MDSCs as CD $11 \mathrm{~b}^{+} \mathrm{CD} 14^{+} \mathrm{HLA}-$ $\mathrm{DR}^{-1 \mathrm{lo}} \mathrm{CD} 15^{-}$. Furthermore, they defined some immature cells as early-stage MDSCs (e-MDSCs) possessing traits common to all MDSCs subsets (16).

MDSCs play an essential role in immunosuppression and possess diverse functions in tumor development, growth, progression and resistance to therapy. They can suppress 


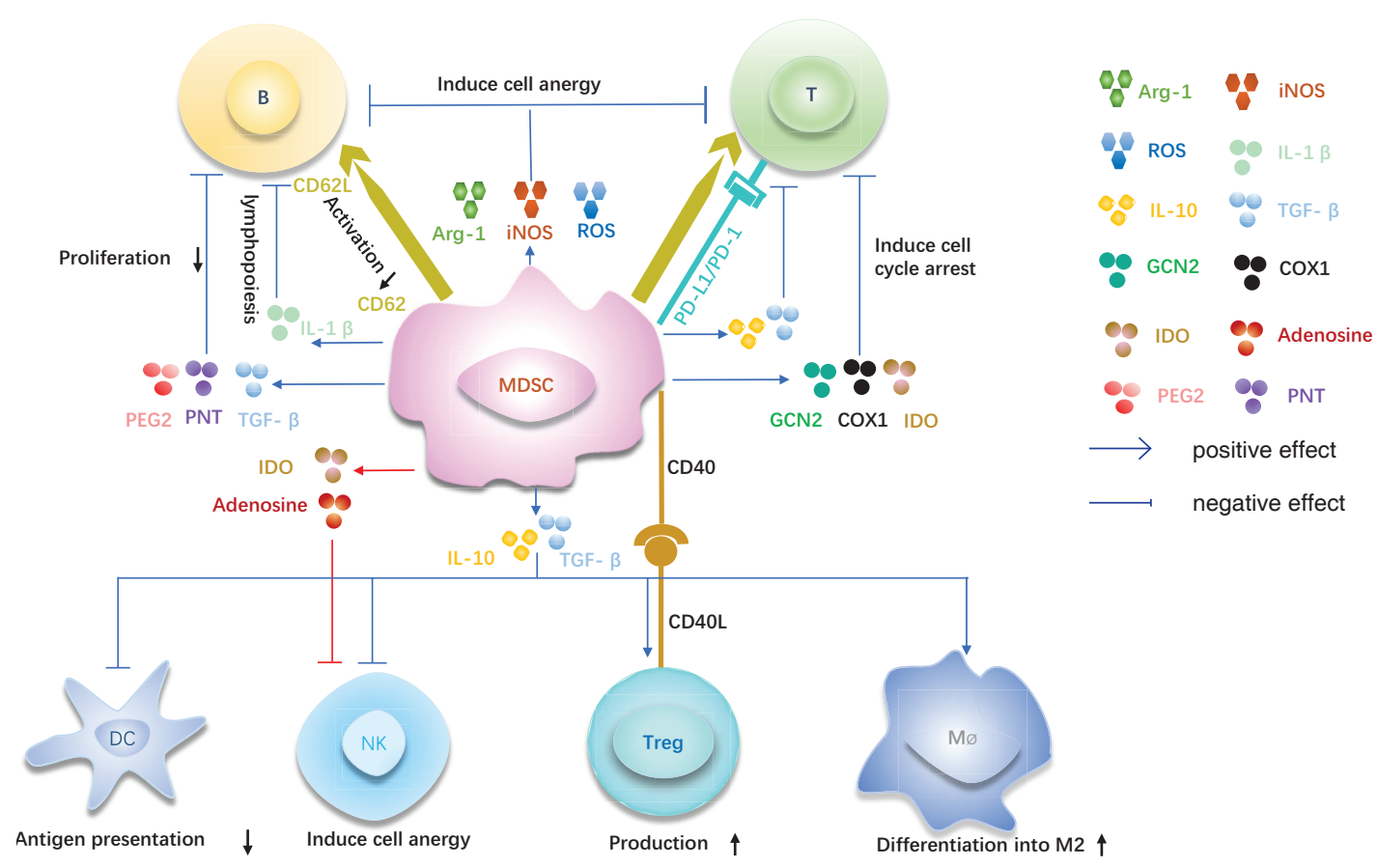

Figure 1 Function of MDSCs. T cell: MDSCs can promote immune suppression by increasing the activity of arginase (Arg-1), iNOS and ROS; high expression of PD-L1 on MDSCs interacts with PD-1 on T cells; MDSCs can downregulate L-selectin (CD62L) and influence T cell function; Indoleamine 2,3 dioxygenase, COX1 and general control nonderepressible 2 will result in immune suppression; Transforming growth factor- $\beta$ and interleukin-10 can produce direct immunosuppressive effect on T cells. B cell: MDSCs inhibit B cell proliferation and function by Arg-1, iNOS, ROS, PNT, PEG2 and TGF- $\beta$; the secretion of interleukin $1 \beta$ inhibits B lymphopoiesis; MDSCs can downregulates CD62L and influence B cell lymphopoiesis. DCs: TGF- $\beta$ and IL-10 inhibit DC the function of antigen presentation. NK: TGF- $\beta$, IL-10, IDO and adenosine induce NK anergy. Tregs: the expression of CD40 on MDSCs induce Tregs accumulation; TGF- $\beta$ and IL-10 increase the production. M ø: TGF- $\beta$ and IL-10 exacerbate macrophage polarize towards to the M2 phenotype. MDSCs, myeloid-derived suppressor cells; ROS, reactive oxygen species; PD-L1, programmed death-ligand 1; PNT, peroxynitrite; DCs, dendritic cell; NK, natural killer cell; Tregs, regulatory cells; $M$ ø, macrophage.

T cell function and promote the expansion of regulatory cells (Tregs) and M2 macrophages. MDSCs downregulate the function of dendritic cells (DCs) and natural killer (NK) cells. Furthermore, MDSCs play a potent suppressive function via suppressing B cell function (17-32).

\section{Mechanisms of MDSC-mediated immune suppression}

In tumor microenvironments, MDSCs play a role in antitumor response and provide a microenvironment for tumor growth via multiple mechanisms (Figure 1). MDSCs promote immune suppression by increasing the activity of arginase (Arg-1) and nitric oxide synthase (iNOS) (17). Arginine is essential for $\mathrm{T}$ cell functions, whereas Arg1 can lead to the deprivation of arginine. iNOS produces nitric oxide and can downregulate $\mathrm{T}$ cell receptor $\zeta$ chain expression, which leads to cell cycle arrest (2). MDSCs can also produce reactive oxygen species (ROS), which induce $\mathrm{T}$ cell apoptosis or $\mathrm{T}$ cell receptor nitrosylation (18). Moreover, immune suppression by MDSCs causes a high expression of programmed death-ligand 1 (PD-L1), which interacts with programmed death 1 (PD-1) on T cells (19). Indoleamine-2,3-dioxygenase (IDO), cyclooxygenase (COX1) and general control nonderepressible 2 (GCN2) also result in immune suppression (20-23). Both IDO and GCN2 can induce the degradation of tryptophan and subsequent cell cycle arrest. IDO also can block the function of NK cells (24). Furthermore, MDSCs express a disintegrin and metalloproteinase domain 17 (ADAM17), which downregulates L-selectin (CD62L) and influences T cells to target peripheral lymph nodes (25). The suppressive mechanisms of MDSCs are induced by the secretion 
of transforming growth factor- $\beta$ (TGF- $\beta$ ) and IL-10, which produce direct immunosuppressive effect on $\mathrm{T}$ cells, induce the generation of Tregs, increase macrophage polarization toward the M2 phenotype, suppress the antigen presentation and function of DCs, and inhibit NK cell production of IFN- $\gamma$ (26-30). The expression of the immune stimulatory receptor CD40 on MDSCs plays a role for Treg accumulation (22). Adenosine from MDSCs is a major NK cell suppressive factor that limits IFN- $\gamma$ and TNF- $\alpha$ release (31). Furthermore, MDSCs regulate B cells during inflammatory responses. The expression of Arg-1, iNOS, ROS, peroxynitrite (PNT), prostaglandin E2 (PEG2) and TGF- $\beta$ inhibit $B$ cell proliferation and antibody production. The secretion of interleukin $1 \beta$ (IL-1 $\beta$ ) inhibits B lymphopoiesis. CD62L B cells can be downregulated by MDSCs, which inhibit lymphocyte movement to activation sites (32). Overall, MDSCs utilize these different mechanisms to mediate immune suppression.

\section{Antitumor effects of targeted MDSCs}

MDSCs play an important role in tumor progression; targeted treatments using MDSCs and the destruction of immune suppressive microenvironments can improve therapeutic effects. The inhibition of MDSCs development, infiltration, and function, their differentiation into mature myeloid cells, and the depletion MDSCs can be tailored to improve treatment effects.

\section{Inhibition of MDSCs development and infiltration}

Signal transducer and activator of transcription 3 (STAT3) plays an essential role in the expansion of MDSCs (33). Curcumin has the ability to inhibit STAT3 targets and can reduce the expression of STAT3 target genes, leading to caspase-dependent apoptosis and mitochondrial membrane potential reduction. More importantly, curcumin does not adversely affect the function of immunostimulatory cytokines, such as granzyme b, IFN- $\gamma$ and IL-2 (34). The expansion of MDSCs, the activation of Stat 3 and NF- $\kappa B$ signals and the secretion of IL- 6 could be inhibited by curcumin treatment (35). Further, galiellalactone, a STAT3 inhibitor, can prevent the generation of MDSCs in prostate cancer, which indicates a promising therapeutic approach (36). Therefore, curcumin treatment may be a strategy for cancer therapy. Flubendazole is used as an anthelmintic and has the ability to block tumor growth via STAT3. Flubendazole can reduce MDSCs levels in tumor tissues and acts as a novel small molecule inhibitor for melanomas (37). Past studies have demonstrated that targeting STAT3 may reverse the immunosuppressive mechanisms caused by MDSCs.

Vascular endothelial growth factor (VEGF) is immunosuppressive. Patients can benefit from VEGF blockades. Sunitinib is a small molecule that can inhibit VEGFR, PDGFR, fetal liver tyrosine kinase 3 and c-Kit. Sunitinib could constrain M-MDSCs and cause the apoptosis of PMN-MDSCs $(38,39)$. Sunitinib has been shown to deplete MDSCs and act synergistically with the $\mathrm{HPV}$ vaccine resulting in enhanced levels of active tumorantigen specific CTLs and altered antitumor immunity (40). Patients treated with sunitinib showed significant reductions in MDSCs, which corresponded with a reversal of type 1 T-cell suppression (41). Bevacizumab is an antiVEGF antibody; when treated with bevacizumab-based chemotherapy, PMN-MDSCs levels significantly reduced, compared with non-bevacizumab-based regimens (42). These studies showed that angiogenesis inhibitors create favorable antitumor microenvironments.

Semaphorin 4D (Sema4D) is a transmembrane glycoprotein and proangiogenic cytokine produced by tumor cells. In human and animal models, Sema4D induced tumor angiogenesis, invasiveness and progression. In human head and neck squamous cell carcinoma cell (HNSCC) lines, Sema4D polarized myeloid cells into an MDSCs phenotype. The use of anti-Sema4D in HNSCC conditional medium significantly reduced the expansion of MDSCs populations (43). VX15/203 binds specifically to Sema4D, which is a humanized IgG4 mAb. This demonstrated an expected pharmacodynamic effect and good clinical therapeutic results in a study of patients with advanced solid tumors (44). Thus, the targeting of Sema4D is also a choice for enhancing antitumor responses.

In patients with melanoma, oncogenic BRAF inhibitor PLX4032 (Vemurafenib) improved the overall survival (45). In another study, M-MDSCs and PMN-MDSCs frequency reduced with Vemurafenib treatment (46).

The chemokine receptor CCR2 is expressed in monocyte-derived MDSCs. Targeting CCR2 expressing cells would enhance immunotherapy effects (47). CCR $5^{+}$ MDSCs and CCR5 ligands would accumulate in melanoma lesions in mice and humans. Tumor-infiltrating CCR5 MDSCs displayed lower immunosuppressive activity than CCR $5^{+}$MDSCs. More importantly, targeting CCR5/ CCR5 ligand interactions indicates better survival, which demonstrates an important role for CCR5 in MDSCs and suggests a novel strategy for cancer treatment (48). 


\section{Differentiation of MDSCs into mature myeloid cells}

$1 \alpha 25(\mathrm{OH})_{2} \mathrm{D}_{3}$ is known as calcitriol. Vitamin $\mathrm{D}$ can result in the terminal differentiation of myeloid cells. Studies have found that vitamin $\mathrm{D}$ induced the monocytic maturation of cell lines (49). When HNSCC patients were treated with vitamin $\mathrm{D}$, the number of $\mathrm{CD} 34^{+}$cells decreased, whereas plasma levels of IL-12 and IFN- $\gamma$ increased, improving T cell blastogenesis (50). In early HNSCC, human MDSCs were characterized by CD34 expression (51). In other clinical trials, 16 HNSCC patients were treated with vitamin $\mathrm{D}$ and achieved increase levels of $\mathrm{CD} 4^{+} \mathrm{T}$ cells and $\mathrm{CD}^{+} \mathrm{T}$ cells. The results also showed that the levels of CD69-positive cells were approximately 10 -fold greater in patients who received vitamin D treatment. Lymphocytes, NK cells and monocytes can express CD69. Groups not receiving vitamin $\mathrm{D}$ treatment before surgery had worse survival rates than groups receiving vitamin D (52-54). However, they did not clarify the mechanism of the immune modulatory effect, because both vitamin $\mathrm{D}$ and vitamin $\mathrm{D}$ analogs may have this function. Besides, the number of patients included in the study was small, requiring further confirmation of clinical effects.

All-trans retinoic acid (ATRA) is a member of the retinoid family and is structurally related to vitamin A. ATRA has been reported as having potent activity in enhancing the differentiation of immature myeloid cells into macrophages, dendritic cells and granulocytes (55). ROS is one of the major factors contributing toward preventing the differentiation of MDSCs into mature myeloid cells (56). According to a study by Nefedova and coworkers, ATRA dramatically upregulated the protein levels of glutathione synthase (GSS), which resulted in the accumulation of glutathione $(\mathrm{GSH})$ via activation of extracellular signalregulated kinase 1/2. GSH can reduce ROS levels and enhance the differentiation of MDSCs (57). The vascular endothelial growth factor receptor-2 (VEGFR-2) is overexpressed in breast cancer. VEGFR-2 has been targeted in clinical practice. The VEGFR-2 blockade antibody ramucirumab did not meaningfully improve important clinical outcomes, and anti-angiogenic therapies have been limited because MDSCs conferred resistance, resulting in detrimental mediators accumulating in tumors (58-60). To address this issue, Bauer et al. combined anti-angiogenic therapies and ATRA. In 4T1 and TS/A mice models, the combination of ATRA and DC101, a monoclonal antibody targeting murine VEGFR-2, reduced tumor volumes and weights compared with DC101 monotherapy. In another study, DC101-induced hypoxia was alleviated by ATRA, which also abrogated the accumulation of MDSCs and simultaneously promoted tumor vessel normalization and maturation (61). The genetic engineering of immune cells has been a novel and promising means for targeted therapy, such as targeting $\mathrm{T}$ cells to generate chimeric antigen receptor-T cells (CAR-T cells) $(62,63)$. In neuroblastoma, CARs targeting the disialoganglioside GD2 present modest antitumor activity $(64,65)$. However, the antitumor activity was limited against osteosarcomas, which resulted in a significant expansion of MDSCs (66). ATRA treatment reduced the number and suppressive capacity of MDSCs; antitumor effects could be enhanced with a combination treatment of ATRA and GD2-CRA T cell therapy. MartireGreco et al. used a lipopolysaccharide-immunosuppressed mice model to study the effects of ATRA on different immune parameters. They demonstrated that ATRA could induce $\mathrm{T}$ cell proliferation and decrease MDSCs numbers, thereby showing a potential treatment of immunosuppressive state patients (67). Furthermore, according to a randomized phase II clinical trial treating stage 3 and stage 4 melanoma patients with ipilimumab alone or with ATRA, ATRA reduced the PD-L1, IL-10 and indoleamine 2,3-dioxygenase gene expression by MDSCs. Compared with ipilimumab treatment alone, ATRA notably decreased the level of circulating MDSCs and improved $\mathrm{CD}^{+} \mathrm{T}$ responses (68). In summary, these studies demonstrated that combinations of ATRA with other treatments can enhance the efficacy of immune treatments.

Most $\beta$-glucans are generated from yeast, bacteria, barley and fungi; the beneficial properties of $\beta$-glucans have long been reported. They have a linear $\beta-1,3-$ linked $\mathrm{D}$-glucose molecule ( $\beta$-1,3-D-glucan) backbone or $\beta-1,6$-linked side chains of $\beta-1,3-D$-glucans of various sizes (69). $\beta$-glucans have the ability to bridge innate and adaptive antitumor immunity and have been considered as adjuvants for tumor immunotherapy. According to a study by Qi et al., the C-type lectin receptor dectin-1 pathway is necessary for yeast-derived particulate $\beta$-glucan activated dendritic cells and macrophages. In the model of ovalbumin-transfected murine mammary carcinoma mice, $\beta$-glucan enhanced antitumor immune responses and drastically downregulated immunosuppressive cells via Th1 and cytotoxic T-lymphocyte priming and differentiation $(70,71)$. To further explore whether $\beta$-glucan could reduce and modify MDSCs function or promote MDSCs differentiation through the dectin-1 pathway, researchers have investigated the effects of $\beta$-glucan treatment on MDSCs in vitro and in tumor models (72). They found that 
the proportion of MDSCs was significantly reduced and a proportion of $\mathrm{CD} 11 \mathrm{c}^{+} \mathrm{F} 4 / 80^{+}$cells emerged with $\beta$-glucan treatment. Moreover, $\mathrm{CD} 11 \mathrm{c}^{+} \mathrm{F} 4 / 80^{+}$cells were more mature than $\mathrm{CD} 11 \mathrm{c}^{-} \mathrm{F} 4 / 80^{-}$cells because the expression of Ly6C was lower in $\mathrm{CD} 11 \mathrm{c}^{+} \mathrm{F} 4 / 80^{+}$. Albeituni et al. found that $\beta$-glucan-treated M-MDSCs could differentiate into $\mathrm{F} 4 / 80^{+} \mathrm{CD} 11 \mathrm{c}^{+}$cells and acted as potent APCs to induce $\mathrm{Ag}$-specific $\mathrm{CD}^{+}$and $\mathrm{CD} 8^{+} \mathrm{T}$ cell responses in a dectin1 -dependent manner (73). To further assess the function of $\beta$-glucan, researchers treated NSCLC patients with $\beta$-glucan and compared the frequency of HLA ${ }^{-} \mathrm{DR}^{-} \mathrm{CD} 14^{-} \mathrm{CD} 11 \mathrm{~b}^{+}$ $\mathrm{CD}_{3} 3^{+}$MDSCs in peripheral blood with controls. They found that the frequency of HLA ${ }^{-} \mathrm{DR}^{-} \mathrm{CD} 14^{-} \mathrm{CD} 11 \mathrm{~b}^{+}$ $\mathrm{CD}_{33^{+}}$MDSCs decreased in the experimental group (73). Overall, combining $\beta$-glucan with other immunotherapeutic approaches may have clinical benefits.

Lactoferrin (LF) is a glycosylated globular protein and is known as the "red protein". It has multifunctional capacity, including immunomodulatory properties, antimicrobial capacity, and antioxidant, anti-inflammatory and anticarcinogenic activity $(74,75)$. LF can modulate the immune microenvironments to prevent metastasis. According to a study by Wei et al., LF deficiency facilitated melanoma cells metastasis. In the tumor metastasis model of LF knockout $\left(\mathrm{LF}^{--}\right)$mice, the level of PMN-MDSCs was upregulated in tumor tissues. When LF was added, the proportion of MDSCs decreased. More importantly, the proportions of macrophages and DCs increased, which demonstrated that LF could regulate the differentiation of MDSCs (76). LF may act in preventing or treating tumor metastasis; however, further research in humans is warranted.

\section{Inhibition of MDSCs function}

AMP-activated protein kinase (AMPK) acts as the main sensor of cellular energy and is conserved across all eukaryotic species. AMPK has been pursued as a therapeutic target of several diseases, such as diabetes, obesity, inflammation and cancer (77). AMPK can regulate the immunosuppressive activity of tumor-MDSCs. In the Lewis lung carcinoma bearing mice model, the AMPK inhibitor dorsomorphin-compound delayed tumor growth and altered MDSCs functions, such as impairing immunosuppressive activity and diminishing expression of Arg-1. However, a higher immunoregulatory response was observed when mice were pretreated with 5 -aminoimidazole-4-carboxamide $1-\beta$-D-ribofuranoside, an AMPK agonist. Thus, the inhibition of AMPK may be a potential therapeutic strategy in cancer (70). In the tumor model, MCA-38, EL4, B16 and MMTV-PyMT tumor volumes presented delayed growths in Prkaa ${ }^{\mathrm{KO}}$ (conditional deletion of the AMPK gene) mice compared with Prkaa $1^{\text {flox }}$. Both PMN-MDSCs and M-MDSCs showed lower immunosuppressive activity from tumors in Prkaa $1^{\mathrm{KO}}$ mice, which further demonstrated the role of AMPK in regulating MDSC-induced immunosuppressive effects (78).

Metformin is prescribed for type 2 diabetes and can benefit hyperinsulinemia and insulin resistance (79). Metformin has been associated with lower risks of cancer (80). In ovarian cancer patients, in peripheral blood and tumor tissues, MDSCs showed elevated expressions of functional CD39/CD73, which significantly contributes to immune suppression through mediating ectoenzymatic activity $(81,82)$. When MDSCs subsets were treated with metformin, reduced expression levels of CD39/CD73 were observed. In a randomized trial, patients receiving metformin showed decreased percentages of $\mathrm{CD} 39^{+}$or $\mathrm{CD} 3^{+} \mathrm{MDSCs}$ and received better prognoses compared with controls (82). However, only cancer patients with diabetes were suitable candidates.

Phosphodiesterase-5 (PDE5) inhibitors, such as sildenafil, can induce apoptosis in chronic lymphocytic leukemia cells; more studies are warranted in vivo (83). PDE5 can also limit MDSCs suppressive activities (84). In C26GM or 4T1-HA tumor mice models, tumor size was smaller in sildenafiltreated groups compared with control groups. Tumor-bearing mice were treated with or without sildenafil. Both Arg1 and NOS2 from intratumoral MDSCs expression were reduced in the sildenafil group. Therefore, PDE5 inhibition abrogated MDSCs suppressive activity. More importantly, PDE5 inhibition can restore T cell proliferation in HNSCC patients (84). In a randomized, prospective, double blinded and placebo controlled clinical trial, a total of 40 HNSCC patients were enrolled and divided into a placebo group and a tadalafiltreated group. Both Arg-1 and iNOS activity were significantly reduced in the tadalafil-treated group compared with controls. The study further demonstrated that PDE5 inhibitor augmented tumor-specific immunity in HNSCC patients (85). Additionally, according to a pilot trial, tadalafil showed biological activity in metastatic melanoma patients. Twenty-five percent of patients achieved a stable disease state; $\mathrm{NO}$ production by M-MDSCs was reduced in the tadalafil-treated group (86). In summary, PDE5 has been shown to reverse tumor-specific immune suppression; however, low patient numbers and shortterm observations necessitates further investigation. 
V-domain immunoglobulin suppressor of $\mathrm{T}$ cell activation (VISTA) is a negative checkpoint. It is homologous to the PD-L1 ligand and belongs in the B7 family (87). In a B16OVA melanoma mice model, VISTA antibody treatment suppressed tumor growth and altered the tumor microenvironment, decreasing M-MDSCs levels and increasing DC levels (88). Corzo et al. investigated the regulation of VISTA by tumor microenvironment factors. Antitumor effects can be suppressed by hypoxia due to the upregulation of suppressive mediators Arg-1 and iNOS. Hypoxia-inducible factor (HIF) $1 \alpha$ is also responsible for MDSCs function (89). In a CT26 murine colon cancer model, VISTA was preferentially upregulated in hypoxic areas (90). VISTA-knockout mice were evaluated for the function of VISTA expression on MDSCs. Under normoxic conditions, MDSCs isolated from VISTA-knockout mice did not influence T-cell suppression, whereas MDSCs suppression was reduced under hypoxic conditions compared with MDSCs from wild type mice (90). T-cell proliferation and activation were upregulated in the presence of antiVISTA antibodies under hypoxia. This demonstrated that hypoxia-induced VISTA promoted the suppressive function of MDSCs in the tumor microenvironment (90). These studies suggested that therapeutic strategies targeting VISTA may have applications in cancer immunotherapy.

Synthetic oligodeoxynucleotides (ODNs) that contain immunostimulatory $\mathrm{CpG}$ motifs trigger immunomodulatory cascades. Clinical studies have demonstrated that $\mathrm{CpG}$ ODNs modulate immune responses and are safe in humans (91). CpG ODNs can contribute to tumor regression via reducing the immunosuppressive activity of MDSCs (92). In a CT26 tumor mice model, intratumoral injections of $\mathrm{CpG}$ ODNs reduced tumor growth. $\mathrm{CpG}$ ODN-treated M-MDSCs did not inhibit T cell activation, and the suppressive activity of M-MDSCs was not influenced with control ODN. Other studies showed that $\mathrm{CpG}$ ODN treatment reduced the production of $\mathrm{NO}$ and Arg-1 by M-MDSCs, thereby inhibiting the suppressive activity of M-MDSCs (92). However, future clinical trials are required to understand $\mathrm{CpG}$ ODN efficacy.

Hydrogen sulfide $\left(\mathrm{H}_{2} \mathrm{~S}\right)$ plays an essential role in many physiological and pathological processes (93). Acetyl deacylasadisulfide (ADA), a vinyl disulfide compound and a new $\mathrm{H}_{2} \mathrm{~S}$-donor, has shown the ability to suppress the proliferation of human melanoma cell lines (94). Thus, a new class of drugs may be generated from ADA in the future. L-Arginine is a precursor of $\mathrm{H}_{2} \mathrm{~S}$; the production of Arg-1 promotes the depletion of L-arginine (2). Corzo et al. demonstrated a novel role for $\mathrm{H}_{2} \mathrm{~S}$ in the modulation of MDSCs in tumors (89). In a B16 tumor model, diallyl trisulfide (DATS), a $\mathrm{H}_{2} \mathrm{~S}$-donor, inhibited tumor growth and upregulated $\mathrm{H}_{2} \mathrm{~S}$ production. M-MDSCs and PMNMDSCs were significantly reduced in the spleen. The expression of MDSC-immunosuppressive genes from DATS-treated mice were lower when compared with controls. T-cell proliferation activity was enhanced when MDSCs from DATS-treated mice were cultured compared with controls. However, this study did not clarify the mechanisms of garlic-derived allyl sulfides (95). Further studies are required to confirm their effects.

\section{Depletion MDSCs}

The therapeutic depletion of MDSCs represents an attractive approach to cancer immunotherapy. Qin et al. synthesized a new therapeutic peptide that specifically binds MDSCs (96). They selected H6 and G3 peptides and fused sequences encoding H6 and G3 with a sequence encoding Fc portion. In a model of EL4 tumor-bearing mice, these peptide bodies depleted M-MDSCs and PMN-MDSCs and repressed tumor development. DCs, T, B and NK cells were not affected (96). It is an effective approach to depleting MDSCs via antibodies against MDSC-specific markers. In a Lewis lung carcinoma-bearing mice model, anti-Gr1 or anti-Ly6G enhanced antitumor activity. The activity of APC, NK and T cells were upregulated. Tumor growth was significantly inhibited, and tumor cell apoptosis was enhanced. Overall, these peptide bodies successfully depleted MDSCs levels. However, this technology has yet to be tested for human MDSCs (97). Kumar et al. hold a different idea. The antitumor efficacy of anti-Gr1 antibody has not been demonstrated. When mice were treated with Gr-1 antibody, the number of PMN-MDSCs decreased but had little effect on M-MDSCs and did not influence tumor growth (98). Therefore, more study is necessary to study the efficacy of antibodies against MDSC-specific markers.

Some studies have reported that some cytotoxic anticancer agents play roles in depleting MDSCs. In a model of EL4 tumor-bearing mice, after injecting 5 -fluorouracil (5FU), MDSCs levels in spleen and tumor beds were significantly reduced compared with controls. Other studies found that 5FU triggered MDSCs apoptosis and induced the activation of $\mathrm{CD} 8^{+} \mathrm{T}$ cells. These authors hypothesized that $5 \mathrm{FU}$ acted as antitumor agents via selective action on MDSCs (99). Additionally, gemcitabine selectively depleted MDSCs in tumor-bearing 
mice and did not reduce the number of $\mathrm{CD}^{+} \mathrm{T}$ cells, $\mathrm{CD}^{+} \mathrm{T}$ cells, NK cells, macrophages and B cells (100). Wang et al. concentrated on the antitumor efficacy of gemcitabine combined with immunotherapy. A clinical study used gemcitabine combined with cytokine-induced killer cells (CIK) or CIK alone to treat 53 solid tumor patients. The combination treatment significantly improved the prognosis of metastatic renal cell carcinoma and advanced pancreatic cancer patients, whereas the prognosis did not improve in metastatic melanoma patients (101). However, chemotherapy may be related to dose-dependent toxicities. Sasso et al. exploited nanoparticulates comprising lipid nanocapsules loaded with a lauroyl-modified form of gemcitabine. This drug reduced the percentage of M-MDSCs in tumor-bearing mice compared with free gemcitabine treatment. These nanoparticulate chemotherapy drug formulations have potential applications (102).

Tyrosine kinase inhibitors have MDSC-targeting abilities. Ibrutinib is an irreversible inhibitor of Bruton's tyrosine kinase and is used for the treatment of B-cell malignancies. According to a study by Stiff et al., ibrutinib could inhibit the generation of human MDSCs in vitro and lead to significant reductions of mice MDSCs in vivo (103). SRC family kinases (SFKs), a group of nonreceptor tyrosine kinases, can also modulate cellular functions. Dasatinib inhibited SFK activity and reduced tumor volume and population of MDSCs in HNSCC mouse models (104). Additionally, a combined treatment of anti-mouse cytotoxic T-lymphocyte antigen 4 and dasatinib significantly reduced the number of MDSCs and tumor volume. In brief, targeting tyrosine kinase is a potential strategy for immune therapy (105).

\section{Conclusions}

At present, MDSCs are one of the reasons leading to poor therapeutic effects in the treatment of many tumors. MDSCs promote tumor growth by various mechanisms; as such, more studies have focused on targeting MDSCs. This review has listed recent methods used for targeting MDSCs to improve anticancer immune responses. However, many studies have been limited to animal experiments. More clinical studies are required to show any potential efficacy. The targeting of MDSCs is an essential and promising method for tumor treatment.

\section{Acknowledgments}

Funding: None.

\section{Footnote}

Provenance and Peer Review: This article was commissioned by the Guest Editor (Jia Wei) for the series "Tumor immune microenvironment in cancer progression and cancer therapy" published in Translational Cancer Research. The article was sent for external peer review organized by the Guest Editor and the editorial office.

Conflicts of Interest: All authors have completed the ICMJE uniform disclosure form (available at http://dx.doi. org/10.21037/tcr.2020.01.52). The series "Tumor immune microenvironment in cancer progression and cancer therapy" was commissioned by the editorial office without any funding or sponsorship. The authors have no other conflicts of interest to declare.

Ethical Statement: The authors are accountable for all aspects of the work in ensuring that questions related to the accuracy or integrity of any part of the work are appropriately investigated and resolved.

Open Access Statement: This is an Open Access article distributed in accordance with the Creative Commons Attribution-NonCommercial-NoDerivs 4.0 International License (CC BY-NC-ND 4.0), which permits the noncommercial replication and distribution of the article with the strict proviso that no changes or edits are made and the original work is properly cited (including links to both the formal publication through the relevant DOI and the license). See: https://creativecommons.org/ licenses/by-nc-nd/4.0/.

\section{References}

1. Groth $\mathrm{C}, \mathrm{Hu} \mathrm{X}$, Weber R, et al. Immunosuppression mediated by myeloid-derived suppressor cells (MDSCs) during tumour progression. Br J Cancer 2019;120:16-25.

2. Consonni FM, Porta C, Marino A, et al. Myeloid-Derived Suppressor Cells: Ductile Targets in Disease. Front Immunol 2019;10:949.

3. Gabrilovich DI, Bronte V, Chen SH, et al. The terminology issue for myeloid-derived suppressor cells. Cancer Res 2007;67:425-6.

4. Jia Y, Li X, Jiang T, et al. EGFR-targeted therapy alters the tumor microenvironment in EGFR-driven lung tumors: Implications for combination therapies. Int J Cancer 2019;145:1432-44. 
5. Kim IS, Gao Y, Welte T, et al. Immuno-subtyping of breast cancer reveals distinct myeloid cell profiles and immunotherapy resistance mechanisms. Nat Cell Biol 2019;21:1113-26.

6. Lu C, Rong D, Zhang B, et al. Current perspectives on the immunosuppressive tumor microenvironment in hepatocellular carcinoma: challenges and opportunities. Mol Cancer 2019;18:130.

7. Marzagalli M, Ebelt ND, Manuel ER. Unraveling the crosstalk between melanoma and immune cells in the tumor microenvironment. Semin Cancer Biol 2019;59:236-50.

8. Ai L, Mu S, Sun C, et al. Myeloid-derived suppressor cells endow stem-like qualities to multiple myeloma cells by inducing piRNA-823 expression and DNMT3B activation. Mol Cancer 2019;18:88.

9. Orillion A, Hashimoto A, Damayanti N, et al. Entinostat Neutralizes Myeloid-Derived Suppressor Cells and Enhances the Antitumor Effect of PD-1 Inhibition in Murine Models of Lung and Renal Cell Carcinoma. Clin Cancer Res 2017;23:5187-201.

10. Mohammadpour H, Bucsek MJ, Hylander BL, et al. Depression Stresses the Immune Response and Promotes Prostate Cancer Growth. Clin Cancer Res 2019;25:2363-5.

11. Lin Q, Ren L, Jian M, et al. The mechanism of the premetastatic niche facilitating colorectal cancer liver metastasis generated from myeloid-derived suppressor cells induced by the S1PR1-STAT3 signaling pathway. Cell Death Dis 2019;10:693.

12. Parker KH, Beury DW, Ostrand-Rosenberg S. MyeloidDerived Suppressor Cells: Critical Cells Driving Immune Suppression in the Tumor Microenvironment. Adv Cancer Res 2015;128:95-139.

13. Tcyganov E, Mastio J, Chen E, et al. Plasticity of myeloidderived suppressor cells in cancer. Curr Opin Immunol 2018;51:76-82.

14. Choi HS, Ha SY,Kim HM, et al. The prognostic effects of tumor infiltrating regulatory $\mathrm{T}$ cells and myeloid derived suppressor cells assessed by multicolor flow cytometry in gastric cancer patients. Oncotarget 2016;7:7940-51.

15. Zhang S, Ma X, Zhu C, et al. The Role of MyeloidDerived Suppressor Cells in Patients with Solid Tumors: A Meta-Analysis. PLoS One 2016;11:e0164514.

16. Bronte V, Brandau S, Chen SH, et al. Recommendations for myeloid-derived suppressor cell nomenclature and characterization standards. Nat Commun 2016;7:12150.

17. Lorenzo-Sanz L, Muñoz P. Tumor-Infiltrating Immunosuppressive Cells in Cancer-Cell Plasticity, Tumor
Progression and Therapy Response. Cancer Microenviron 2019;12:119-32.

18. Nagaraj S, Gupta K, Pisarev V, et al. Altered recognition of antigen is a mechanism of CD8+ T cell tolerance in cancer. Nat Med 2007;13:828-35.

19. Dieterich LC, Ikenberg K, Cetintas T, et al. TumorAssociated Lymphatic Vessels Upregulate PDL1 to Inhibit T-Cell Activation. Front Immunol 2017;8:66.

20. Yu J, Du W, Yan F, et al. Myeloid-derived suppressor cells suppress antitumor immune responses through IDO expression and correlate with lymph node metastasis in patients with breast cancer. J Immunol 2013;190:3783-97.

21. Shi M, Shi G, Tang J, et al. Myeloid-derived suppressor cell function is diminished in aspirin-triggered allergic airway hyperresponsiveness in mice. J Allergy Clin Immunol 2014;134:1163-74.e16.

22. Pan PY, Ma G, Weber KJ, et al. Immune stimulatory receptor CD40 is required for $\mathrm{T}$-cell suppression and $\mathrm{T}$ regulatory cell activation mediated by myeloid-derived suppressor cells in cancer. Cancer Res 2010;70:99-108.

23. Platten M, von Knebel Doeberitz N, Oezen I, et al. Cancer Immunotherapy by Targeting IDO1/TDO and Their Downstream Effectors. Front Immunol 2015;5:673.

24. Zhang J, Han X, Hu X, et al. IDO1 impairs NK cell cytotoxicity by decreasing NKG2D/NKG2DLs viapromoting miR-18a. Mol Immunol 2018;103:144-55.

25. Hanson EM, Clements VK, Sinha P, et al. Myeloid-derived suppressor cells down-regulate L-selectin expression on CD4+ and CD8+ T cells. J Immunol 2009;183:937-44.

26. Ostrand-Rosenberg S, Sinha P, Beury DW, et al. Crosstalk between myeloid-derived suppressor cells (MDSC), macrophages, and dendritic cells enhances tumor-induced immune suppression. Semin Cancer Biol 2012;22:275-81.

27. Kwa niak K, Czarnik-Kwa niak J, Maziarz A, et al. Scientific reports concerning the impact of interleukin 4, interleukin 10 and transforming growth factor on cancer cells. Cent Eur J Immunol 2019;44:190-200.

28. Huang B, Pan PY, Li Q, et al. Gr-1+CD115+ immature myeloid suppressor cells mediate the development of tumor-induced $\mathrm{T}$ regulatory cells and T-cell anergy in tumor-bearing host. Cancer Res 2006;66:1123-31.

29. Mittal SK, Roche PA. Suppression of antigen presentation by IL-10. Curr Opin Immunol 2015;34:22-7.

30. Fogel-Petrovic M, Long JA, Misso NL, et al. Physiological concentrations of transforming growth factor beta1 selectivelyinhibit human dendritic cell function. Int Immunopharmacol 2007;7:1924-33.

31. Raskovalova T, Lokshin A, Huang X, et al. Adenosine- 
Mediated Inhibition of Cytotoxic Activity and Cytokine Production by IL-2/NKp46-Activated NK cells. Immunol Res 2006;36:91-9.

32. Özkan B, Lim H, Park SG. Immunomodulatory Function of Myeloid-Derived Suppressor Cells during B CellMediated Immune Responses. Int J Mol Sci 2018; 19:1468.

33. Gabrilovich DI, Nagaraj S. Myeloid-derived suppressor cells as regulators of the immune system. Nat Rev Immunol 2009;9:162-74.

34. Bill MA, Fuchs JR, Li C, et al. The small molecule curcumin analog FLLL32 induces apoptosis in melanoma cells via STAT3 inhibition and retains the cellular response to cytokines with anti-tumor activity. Mol Cancer 2010;9:165.

35. Tu SP, Jin H, Shi JD, et al. Curcumin induces the differentiation of myeloid-derived suppressor cells and inhibits their interaction with cancer cells and related tumor growth. Cancer Prev Res (Phila) 2012;5:205-15.

36. Hellsten R, Lilljebjorn L, Johansson M, et al. The STAT3 inhibitor galiellalactone inhibits the generation of MDSClike monocytes by prostate cancer cells and decreases immunosuppressive and tumorigenic factors. Prostate 2019;79:1611-21.

37. Li Y, Acharya G, Elahy M, et al. The anthelmintic flubendazole blocks human melanoma growth and metastasis and suppresses programmed cell death protein-1 and myeloid-derived suppressor cell accumulation. Cancer Lett 2019;459:268-76.

38. Yang J, Yan J, Liu B. Targeting VEGF/VEGFR to Modulate Antitumor Immunity. Front Immunol 2018;9:978.

39. Santoni M, Conti A, Massari F, et al. Treatment-related fatigue with sorafenib, sunitinib and pazopanib in patients with advanced solid tumors: an up-to-date review and meta-analysis of clinical trials. Int J Cancer 2015;136:1-10.

40. Draghiciu O, Nijman HW, Hoogeboom BN, et al. Sunitinib depletes myeloid-derived suppressor cells and synergizes with a cancer vaccine to enhance antigenspecific immune responses and tumor eradication. Oncoimmunology 2015;4:e989764.

41. Ko JS, Zea AH, Rini BI, et al. Sunitinib mediates reversal of myeloid-derived suppressor cell accumulation in renal cell carcinoma patients. Clin Cancer Res 2009;15:2148-57.

42. Koinis F, Vetsika EK, Aggouraki D, et al. Effect of FirstLine Treatment on Myeloid-Derived Suppressor Cells' Subpopulations in the Peripheral Blood of Patients with Non-Small Cell Lung Cancer. J Thorac Oncol 2016;11:1263-72.
43. Younis RH, Han KL, Webb TJ. Human Head and Neck Squamous Cell Carcinoma-Associated Semaphorin 4D Induces Expansion of Myeloid-Derived Suppressor Cells. J Immunol 2016;196:1419-29.

44. Patnaik A, Weiss GJ, Leonard JE, et al. Safety, Pharmacokinetics, and Pharmacodynamics of a Humanized Anti-Semaphorin 4D Antibody, in a First-In-Human Study of Patients with Advanced Solid Tumors. Clin Cancer Res 2016;22:827-36.

45. Mok S, Tsoi J,Koya RC, et al. Inhibition of colony stimulating factor-1 receptor improves antitumor efficacy of BRAF inhibition. BMC Cancer 2015;15:356.

46. Schilling B, Sucker A, Griewank K, et al. Vemurafenib reverses immunosuppression by myeloid derived suppressor cells. Int J Cancer 2013;133:1653-63.

47. Lesokhin AM, Hohl TM, Kitano S, et al. Monocytic CCR2(+) myeloid-derived suppressor cells promote immune escape by limiting activated CD8 T-cell infiltration into the tumor microenvironment. Cancer Res 2012;72:876-86.

48. Blattner C, Fleming V, Weber R, et al. CCR5(+) MyeloidDerived Suppressor Cells Are Enriched and Activated in Melanoma Lesions. Cancer Res 2018;78:157-67.

49. Testa U, Masciulli R, Tritarelli E, et al. Transforming growth factor-beta potentiates vitamin D3-induced terminal monocytic differentiation of human leukemic cell lines. J Immunol 1993;150:2418-30.

50. Lathers DM, Clark JI, Achille NJ, et al. Phase 1B study to improve immune responses in head and neck cancer patients using escalating doses of 25-hydroxyvitamin D3. Cancer Immunol Immunother 2004;53:422-30.

51. Talmadge JE, Gabrilovich DI. History of myeloid-derived suppressor cells. Nat Rev Cancer 2013;13:739-52.

52. Walsh JE, Clark AM, Day TA, et al. Use of alpha,25dihydroxyvitamin D3 treatment to stimulate immune infiltration into head and neck squamous cell carcinoma. Hum Immunol 2010;71:659-65.

53. López-Albaitero A, Lee SC, Morgan S, et al. Role of polymorphic Fc gamma receptor IIIa and EGFR expression level in cetuximab mediated, NK cell dependent in vitro cytotoxicity of head and neck squamous cell carcinoma cells. Cancer Immunol Immunother 2009;58:1853-64.

54. Freeman CM, Martinez FJ, Han MK, et al. Lung dendritic cell expression of maturation molecules increases with worsening chronic obstructive pulmonary disease. Am J Respir Crit Care Med 2009;180:1179-88.

55. Kusmartsev S, Cheng F, Yu B, et al. All-trans-retinoic acid 
eliminates immature myeloid cells from tumor-bearing mice and improve the effect of vaccination. Cancer Res 2003;63:4441-9.

56. Kusmartsev S, Gabrilovich DI. Inhibition of myeloid cell differentiation in cancer: the role of reactive oxygen species. J Leukoc Biol 2003;74:186-96.

57. Nefedova Y, Fishman M, Sherman S, et al. Mechanism of all-trans retinoic acid effect on tumor-associated myeloidderived suppressor cells. Cancer Res 2007;67:11021-8.

58. Guo S, Colbert LS, Fuller M, et al. Vascular endothelial growth factor receptor-2 in breast cancer. Biochim Biophys Acta 2010;1806:108-21.

59. Mackey JR, Ramos-Vazquez M, Lipatov O, et al. Primary results of ROSE/TRIO-12, a randomized placebocontrolled phase III trial evaluating the addition of ramucirumab to first-line docetaxel chemotherapy in metastatic breast cancer. J Clin Oncol 2015;33:141-8.

60. Shojaei F, Wu X, Malik AK, et al. Tumor refractoriness to anti-VEGF treatment is mediated by CD11b+Gr1+ myeloid cells. Nat Biotechnol 2007;25:911-20.

61. Bauer R, Udonta F, Wroblewski M, et al. Blockade of Myeloid-Derived Suppressor Cell Expansion with All-Trans Retinoic Acid Increases the Efficacy of Antiangiogenic Therapy. Cancer Res 2018;78:3220-32.

62. Lee DW, Barrett DM, Mackall C, et al. The future is now: chimeric antigen receptors as new targeted therapies for childhood cancer. Clin Cancer Res 2012;18:2780-90.

63. Bailey SR, Maus MV. Gene editing for immune cell therapies. Nat Biotechnol 2019;37:1425-34.

64. Pule MA, Savoldo B, Myers GD, et al. Virus-specific T cells engineered to coexpress tumor-specific receptors: persistence and antitumor activity in individuals with neuroblastoma. Nat Med 2008;14:1264-70.

65. Park JR, Digiusto DL, Slovak M, et al. Adoptive transfer of chimeric antigen receptor re-directed cytolytic $\mathrm{T}$ lymphocyte clones in patients with neuroblastoma. Mol Ther 2007;15:825-33.

66. Long AH, Highfill SL, Cui Y, et al. Reduction of MDSCs with All-trans Retinoic Acid Improves CAR Therapy Efficacy for Sarcomas. Cancer Immunol Res 2016;4:869-80.

67. Martire-Greco D, Landoni VI, Chiarella P, et al. all-transretinoic acid improves immunocompetence in a murine model of lipopolysaccharide-induced immunosuppression. Clin Sci (Lond) 2014;126:355-65.

68. Tobin RP, Jordan KR, Robinson WA, et al. Targeting myeloid-derived suppressor cells using all-trans retinoic acid in melanoma patients treated with Ipilimumab. Int Immunopharmacol 2018;63:282-91.
69. Li B, Cai Y, Qi C, et al. Orally administered particulate beta-glucan modulates tumor-capturing dendritic cells and improves antitumor T-cell responses in cancer. Clin Cancer Res 2010;16:5153-64.

70. Qi C, Cai Y, Gunn L, et al. Differential pathways regulating innate and adaptive antitumor immune responses by particulate and soluble yeast-derived betaglucans. Blood 2011;117:6825-36.

71. Goodridge HS, Reyes CN, Becker CA, et al. Activation of the innate immune receptor Dectin-1 upon formation of a 'phagocytic synapse'. Nature 2011;472:471-5.

72. Tian J, Ma J, Ma K, et al. beta-Glucan enhances antitumor immune responses by regulating differentiation and function of monocytic myeloid-derived suppressor cells. Eur J Immunol 2013;43:1220-30.

73. Albeituni SH, Ding C, Liu M, et al. Yeast-Derived Particulate beta-Glucan Treatment Subverts the Suppression of Myeloid-Derived Suppressor Cells (MDSC) by Inducing Polymorphonuclear MDSC Apoptosis and Monocytic MDSC Differentiation to APC in Cancer. J Immunol 2016;196:2167-80.

74. Moreno-Expósito L, Illescas-Montes R, MelguizoRodriguez L, et al. Multifunctional capacity and therapeutic potential of lactoferrin. Life Sci 2018;195:61-4.

75. Wang B, Timilsena YP, Blanch E, et al. Lactoferrin: Structure, function, denaturation and digestion. Crit Rev Food Sci Nutr 2019;59:580-96.

76. Wei L, Zhang X, Wang J, et al. Lactoferrin deficiency induces a pro-metastatic tumor microenvironment through recruiting myeloid-derived suppressor cells in mice. Oncogene 2020;39:122-35.

77. Garcia D, Shaw RJ. AMPK: Mechanisms of Cellular Energy Sensing and Restoration of Metabolic Balance. Mol Cell 2017;66:789-800.

78. Trillo-Tinoco J, Sierra RA, Mohamed E, et al. AMPK Alpha-1 Intrinsically Regulates the Function and Differentiation of Tumor Myeloid-Derived Suppressor Cells. Cancer Res 2019;79:5034-47.

79. Bodmer M, Meier C, Krahenbuhl S, et al. Long-term metformin use is associated with decreased risk of breast cancer. Diabetes Care 2010;33:1304-8.

80. Libby G, Donnelly LA, Donnan PT, et al. New Users of Metformin Are at Low Risk of Incident Cancer: A cohort study among people with type 2 diabetes. Diabetes Care 2009;32:1620-5.

81. Li J, Wang L, Chen X, et al. CD39/CD73 upregulation on myeloid-derived suppressor cells via TGF-beta-mTORHIF-1 signaling in patients with non-small cell lung 
cancer. Oncoimmunology 2017;6:e1320011.

82. Li L, Wang L, Li J, et al. Metformin-Induced Reduction of CD39 and CD73 Blocks Myeloid-Derived Suppressor Cell Activity in Patients with Ovarian Cancer. Cancer Res 2018;78:1779-91.

83. Sarfati M, Mateo V, Baudet S, et al. Sildenafil and vardenafil, types 5 and 6 phosphodiesterase inhibitors, induce caspase-dependent apoptosis of B-chronic lymphocytic leukemia cells. Blood 2003;101:265-9.

84. Serafini P, Meckel K, Kelso M, et al. Phosphodiesterase-5 inhibition augments endogenous antitumor immunity by reducing myeloid-derived suppressor cell function. J Exp Med 2006;203:2691-702.

85. Califano JA, Khan Z, Noonan KA, et al. Tadalafil augments tumor specific immunity in patients with head and neck squamous cell carcinoma. Clin Cancer Res 2015;21:30-8.

86. Hassel JC, Jiang H, Bender C, et al. Tadalafil has biologic activity in human melanoma. Results of a pilot trial with Tadalafil in patients with metastatic Melanoma (TaMe). OncoImmunology 2017;6:e1326440.

87. Wang L, Rubinstein R, Lines JL, et al. VISTA, a novel mouse Ig superfamily ligand that negatively regulates $\mathrm{T}$ cell responses. J Exp Med 2011;208:577-92.

88. Le Mercier I, Chen W, Lines JL, et al. VISTA Regulates the Development of Protective Antitumor Immunity. Cancer Res 2014;74:1933-44.

89. Corzo CA, Condamine T, Lu L, et al. HIF-1alpha regulates function and differentiation of myeloid-derived suppressor cells in the tumor microenvironment. J Exp Med 2010;207:2439-53.

90. Deng J, Li J, Sarde A, et al. Hypoxia-Induced VISTA Promotes the Suppressive Function of Myeloid-Derived Suppressor Cells in the Tumor Microenvironment. Cancer Immunol Res 2019;7:1079-90.

91. Klinman DM. Immunotherapeutic uses of $\mathrm{CpG}$ oligodeoxynucleotides. Nat Rev Immunol 2004;4:249-58.

92. Shirota Y, Shirota H, Klinman DM. Intratumoral injection of $\mathrm{CpG}$ oligonucleotides induces the differentiation and reduces the immunosuppressive activity of myeloid-derived suppressor cells. J Immunol 2012;188:1592-9.

93. Wallace JL, Wang R. Hydrogen sulfide-based therapeutics: exploiting a unique but ubiquitous gasotransmitter. Nat Rev Drug Discov 2015;14:329-45.

94. De Cicco P, Panza E, Armogida C, et al. The Hydrogen Sulfide Releasing Molecule Acetyl Deacylasadisulfide Inhibits Metastatic Melanoma. Front Pharmacol 2017;8:65. 95. De Cicco P, Ercolano G, Rubino V, et al. Modulation of the functions of myeloid-derived suppressor cells: A new strategy of hydrogen sulfide anti-cancer effects. Br J Pharmacol 2020;177:884-97.

96. Qin H, Lerman B, Sakamaki I, et al. Generation of a new therapeutic peptide that depletes myeloid-derived suppressor cells in tumor-bearing mice. Nat Med 2014;20:676-81.

97. Srivastava MK, Zhu L, Harris-White M, et al. Myeloid suppressor cell depletion augments antitumor activity in lung cancer. PLoS One 2012;7:e40677.

98. Kumar V, Cheng P, Condamine T, et al. CD45 Phosphatase Inhibits STAT3 Transcription Factor Activity in Myeloid Cells and Promotes Tumor-Associated Macrophage Differentiation. Immunity 2016;44:303-15.

99. Vincent J, Mignot G, Chalmin F, et al. 5-Fluorouracil selectively kills tumor-associated myeloid-derived suppressor cells resulting in enhanced T cell-dependent antitumor immunity. Cancer Res 2010;70:3052-61.

100.Suzuki E, Kapoor V, Jassar AS, et al. Gemcitabine selectively eliminates splenic Gr-1+/CD11b+ myeloid suppressor cells in tumor-bearing animals and enhances antitumor immune activity. Clin Cancer Res 2005;11:6713-21.

101. Wang Z, Liu Y, Zhang Y, et al. MDSC decreasing chemotherapy increase the efficacy of cytokine induced killer cell immunotherapy in metastatic renal cell carcinoma and pancreatic cancer. Oncotarget 2016;7:4760-9.

102. Sasso MS, Lollo G, Pitorre M, et al. Low dose gemcitabine-loaded lipid nanocapsules target monocytic myeloid-derived suppressor cells and potentiate cancer immunotherapy. Biomaterials 2016;96:47-62.

103. Stiff A, Trikha P, Wesolowski R, et al. Myeloid-Derived Suppressor Cells Express Bruton's Tyrosine Kinase and Can Be Depleted in Tumor-Bearing Hosts by Ibrutinib Treatment. Cancer Res 2016;76:2125-36.

104.Mao L, Deng WW, Yu GT, et al. Inhibition of SRC family kinases reduces myeloid-derived suppressor cells in head and neck cancer. Int J Cancer 2017;140:1173-85.

105. Yu GT, Mao L, Wu L, et al. Inhibition of SRC family kinases facilitates anti-CTLA4 immunotherapy in head and neck squamous cell carcinoma. Cell Mol Life Sci 2018;75:4223-34.

Cite this article as: Zeng $\mathrm{D}$, Long $\mathrm{H}$, Zhu B. Antitumor effects of targeting myeloid-derived suppressive cells. Transl Cancer Res 2020;9(9):5787-5797. doi: 10.21037/tcr.2020.01.52 